\title{
A GENERAL PROVINCIAL SITUATION VISUALIZATION SYSTEM BASED ON IPHONE OPERATING SYSTEM OF SHANDONG PROVINCE
}

\author{
Zi Ye, Hengmao Xiang \\ Shandong Provincial Institute of Land Surveying and Mapping, Jinan, Shandong, 250102, China \\ zye0916@gmail.com \\ hmxiang2003@126.com
}

Commission

KEY WORDS: General Provincial Situation; GIS Visualization; Mobile devices; iOS; iPad

\begin{abstract}
:
The paper discusses the basic principles and the problem solutions during the design and implementation of the mobile GIS system, and base on the research result, we developed the General Provincial Situation Visualization System Based on iOS of Shandong Province. The system is developed in the Objective-C programming language, and use the ArcGIS Runtime SDK for IOS as the development tool to call the 'World-map Shandong' services to implement the development of the General Provincial Situation Visualization System Based on iOS devices. The system is currently available for download in the Appstore and is chosen as the typical application case of ESRI China ArcGIS API for iOS.
\end{abstract}

\section{INTRODUCTION}

The general provincial situation information includes basic geographic information, natural resources information, economic statistics data and social development statistics data. It covers the information of different levels within one province: the provincial level, the city level, the county level, and the township level, etc. with a multi-scale, multi-temporal characteristic

The multi-scale characteristic of the general provincial situation information requires the General Provincial Situation System can not only demonstrate the macro socio-economic profile of the province at the provincial level, but also can provide a detailed socio-economic development data and related information on municipal or county level by scaling down the spatial data. On the other hand, the multi-temporal characteristic of the general provincial situation information decides the continuity and dynamics of the general provincial situation information, which means the General Provincial Situation System needs to integrate the development information of various fields of the social and economic over the last years in one province, and moreover, to continuously demonstrate the historical conditions and trends in the form of a visual data charts.

There had variety of general provincial situation GIS systems been built up during these years, and they integrated in rich information and complex contents. Whereas, people became more and more used to mobile devices in their daily life and work scene in recent years, and the situation is also occurred in government officials. There are some representative mobile operating system such as the iOS of Apple Inc. and the Android operating system of Google. The iOS is popular and influences the mobile application products by its friendlier user interface which is changing people from all aspects of electronic product usage. Under this background, more and more developers join in the development of mobile GIS applications on the iOS or Android. In this situation, as a typical GIS system to reflect the situation in one province from multiple dimensions, the best solution to design and development the general provincial situation visualization system based on mobile operation system is becoming a problems needing to be solved. Relative to the traditional GIS system building on PCs, there are several problems needing to be solved during the design and development of the GIS systems which is built on the mobile operating system, and specifically we take the iOS as example in this paper. The most urgent problems could be the screen size of the mobile device is limited and different, and the operating practices should be changed from the traditional mouse and keyboard operations to the flexible gestures. What's more, the users interact habits and visual design styles of mobile products have various differences to the traditional products on PCs.

\section{PRINCIPLES OF GIS SYSTEM DESIGN ON MOBILE DEVICES}

Mobile devices (tablet PCs, smart phones) in people's work and life had occupied a pivotal position, which is different from the traditional system design on the PC end especially on the user experiences and interactive features layout. The main problems need to be considered in the design of mobile system applications are the limited screen size and different sizes of mobile devices, the variety of user scenarios, more flexible and natural interactive gestures, lack of the unity and norms, and many other issues.

The general provincial situation data of the system is rich and wide-range, which has a strong target for application. Therefore, in order to complete the efficient view and accurate analysis of the general provincial situation statistic data for the mobile device users, we keep the following principles in the design and development of the system:

(1) The text and features should be as concise as possible. Maximize the use buttons, labels, and other touch-screen interactive elements to replace the common used hypertext click responsive controls of the traditional $\mathrm{PC}$, in order to fit the features and interact of the smaller screen size of mobile device; 
(2) A vertical navigation will be more suitable for the mobile application designed. In the situation of a rich content system, the vertical navigation is the best solution to the dilemma of the limited screen size of mobile devices and the need to leave enough space for the gestures;

(3)Adapt the flattening level design in the system. The flatten system is the mainstream trend design for mobile devices, since a deep structural level lead the user to easily get confused in the convention of hierarchy changing, which is the problem prevalent in the traditional Windows software products. The flat design improves friendly interface and effective solution to the hierarchy problem. Therefore, this system uses tabs combined classification to present the content of the general provincial situation information, and limit the click operation to expand the thematic map view hierarchy to just one layer;

(4) Unlike the mouse-based operating system on the PCs, the mobile devices support a rich touch-screen gestures, the most popular eight gestures are the press, touch, slide, drag, rotate, zoom and shake. The general provincial situation visualization system can operate the map data and browse the thematic maps through rich gestures, which is just suitable to the multi-scale and multi-temporal characteristics of the general provincial situation information.

Following the above principles, in the three times' revision process of the system, the buttons of vector/image switching is combined into one button, and the map resetting button and the plot clearing button is combined into one also. We remove the popular shutter menu operation, and allow all the core users operating functions be flatten distributed on the surface of the app's home page. Though the System relate to more than 20 categories of thematic maps and statistical charts, we decided the color, 2D / 3D forms and other characteristics for each chart and map through combined its own characteristic of the data with the common form of thematic charts which is been divided as the symbol-levelled thematic maps, the range-classified thematic maps and the statistical thematic map.

\section{SYSTEM ARCHITECTURE DESIGN}

\subsection{The Data Layer}

The data layer of the General Provincial Situation Visualization System Based on iOS of Shandong Province can be divided as the geo-spatial data and the general provincial statistical data. Both the data covered not only the provincial development data, but also the 17 cities and 140 counties statistical data.

Firstly, the geo-spatial data includes the 1:50000, 1:10000, $1: 5000,1: 1000$ to $1: 500$ scale spatial data. Secondly, the general provincial statistical data includes the demographic data, the economy data and the major planning data in recent years.

In this system, the geo-spatial data is directly called by the service interface 'World-map Shandong' map data service resources through ArcGIS Runtime SDK for IOS. And the general provincial statistical data is stored in the Orcale $10 \mathrm{~g}$ database and released as the general provincial statistical information services through the back-end release system in 'World-map Shandong'. After the release job, the services can be called by the unified interface displayed through the iOS front-end development to show.

\subsection{The Service Layer}

The service layer provides the service interfaces of the General Provincial Situation Visualization System Based on iOS of Shandong Province is designed to comply with the specifications of service release and registration Interface of the 'World-map Shandong'. Those interfaces are packaged as the Web Service, which can shield all the complex heterogeneous data access. Furthermore, since the service interfaces and parameters are be designed as a uniform standard, the application systems can reduce the development cost through achieving the transparent access not regards the detail issues such as the transaction processing and the concurrency control.

\subsection{The Application Layer}

The application layer is designed to meet the users' demand which is focused on the intuitive and efficient expression of the general provincial situation information. It is designed with the Apple's iOS style, and supports the iPad gestures and user habits with a flexible layout to optimize the user interaction and experience.

The General Provincial Situation Visualization System Based on iOS of Shandong Province is composed of three functional modules logically divided: Map View module, chart visualization module and geographic visualization module.

\section{FUNCTION MODULES DESIGN}

\subsection{The Map View Module}

In the Map View module, we use the multi-touch gestures such as swipe, tap, pinch, and reverse pinch to view the map in different scale and complete the query operations.

\subsubsection{Map browsing}

The most frequently used gestures such as swipe, tap, pinch, and reverse pinch to complete the zoom, pan and other common map operation. In addition, the system provides the basic features include clear-up, map plotting, whole-map view, positioning, vector maps and image maps switching, etc., to meet the requirements of the statistics visualization in the system.

\subsubsection{Query function}

The system offers a variety of ways to support the selection query analysis of geographical units, which include the point selection and polygon selection by clicking and sliding gestures on the screen.

\subsubsection{Map plotting}

Map plotting is designed to allow users to gather the information they care about beyond a certain region, and mark the information on the map for an easy search and use in later time. By clicking, paddling and other gestures the plotting can be drawn in points, lines or areas with the relevant text annotations.

\subsection{The Chart Visualization Module}

The Chart visualization module provides seven statistic charts with 2-dimensional or 3-dimensional to complete the users' selection of the chart list and the present of statistical charts information. They are bar chart, line chart, pie chart, area chart, circle chart, rose chart and column chart. These seven statistical 
charts are selected as the most frequently used charts in maprelated statistical information expression.

\subsection{The Geographic Visualization Module}

The geographic visualization module provides three kinds of thematic map to present the content of statistical charts: the symbol-levelled thematic maps, the range-classified thematic maps and the statistical thematic map.

\subsubsection{The Symbol-levelled Thematic Naps}

The symbol-levelled thematic maps use the size of the symbol to represent the density, the intensity or the level of development statistics. By the symbol-levelled thematic maps, users can easily understand the relative statistical relationship between the map areas visually.

\subsubsection{The Range-classified Thematic Maps}

The range-classified thematic maps are commonly used in the productions of various types of economic and social thematic maps. In the general provincial situation visualization system, the differences between the administrative division mapping units can be rendered by the color from shallow to deep or the halo line from sparse to dense, which is based on the density, intensity or level of development of a hierarchy of statistical data.

\subsubsection{The Statistical Thematic Maps}

The statistical thematic maps plot the statistical chart of a fixed location on the corresponding location of the map to indicate the changing of a certain phenomenon. The most commonly used charts are the bar chart, the line charts, the rose charts. Through the three-dimensional design of these charts, the statistical data can be expressed in a more intuitive and vivid way.

\section{THE COMPLETION AND APPLICATION OF THE SHANDONG GENERAL PROVINCIAL SITUATION VISUALIZATION SYSTEM BASED ON IPHONE OPERATING SYSTE}

The system is developed in the Objective-C programming language, and using ArcGIS Runtime SDK for IOS as the development tool to call the 'World-map Shandong' service resources to implement the development job of the General Provincial Situation Visualization System Based on iOS devices.

In the system, we included four types' statistical charts and maps which are the population, economic, Main Functional Area Planning and Major Planning, and there are 20 charts and 13 maps represent the social and economic situation of Shandong province during the last 5 years (Figure 1). Economic statistic data is always be the most concern information for those government officials and institute researches, so we specifically show the economic information charts such as the GNP, China hundred top towns in Shandong province, the regional fiscal income, the per capita income of urban and rural residents, the investment of real estate, input and export value(Figure 2), the high-tech industry output and the Economic development zones (Figure 3). In order to express the detailed information more understandable and visual, we particularly design the score map and top/last 10 countdowns of the regional fiscal income and the per capita income of urban and rural residents (Figure 4).
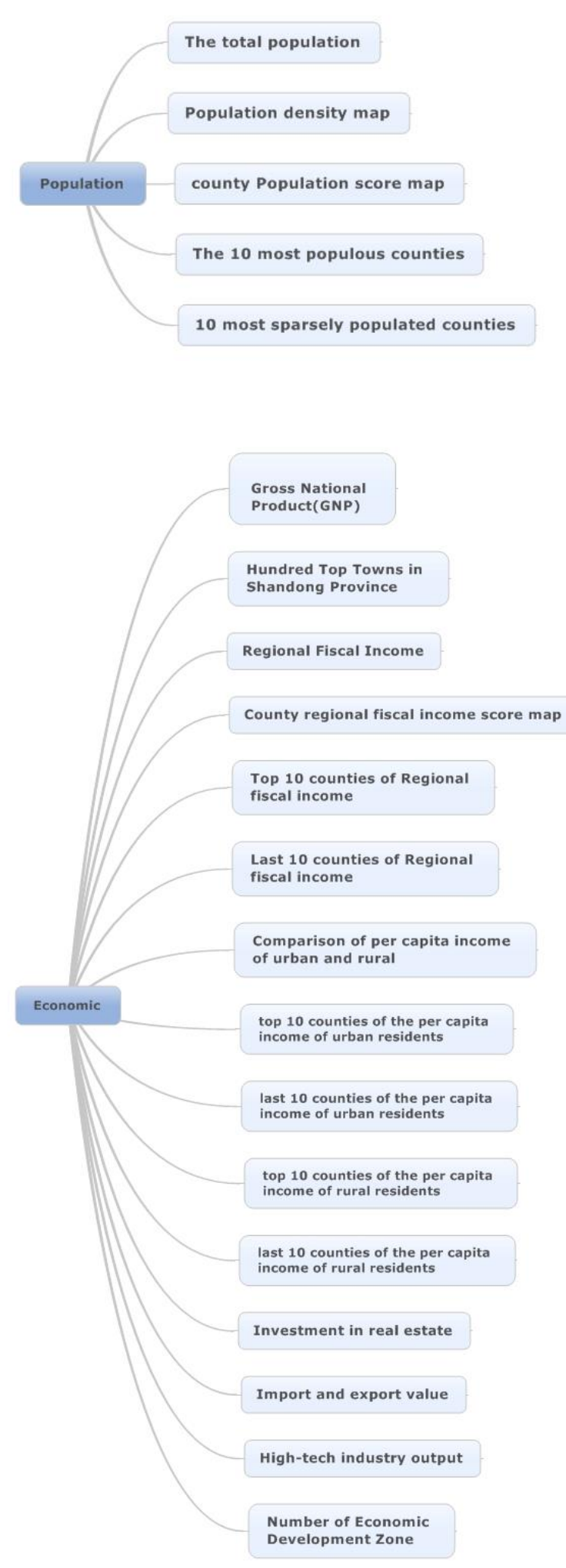

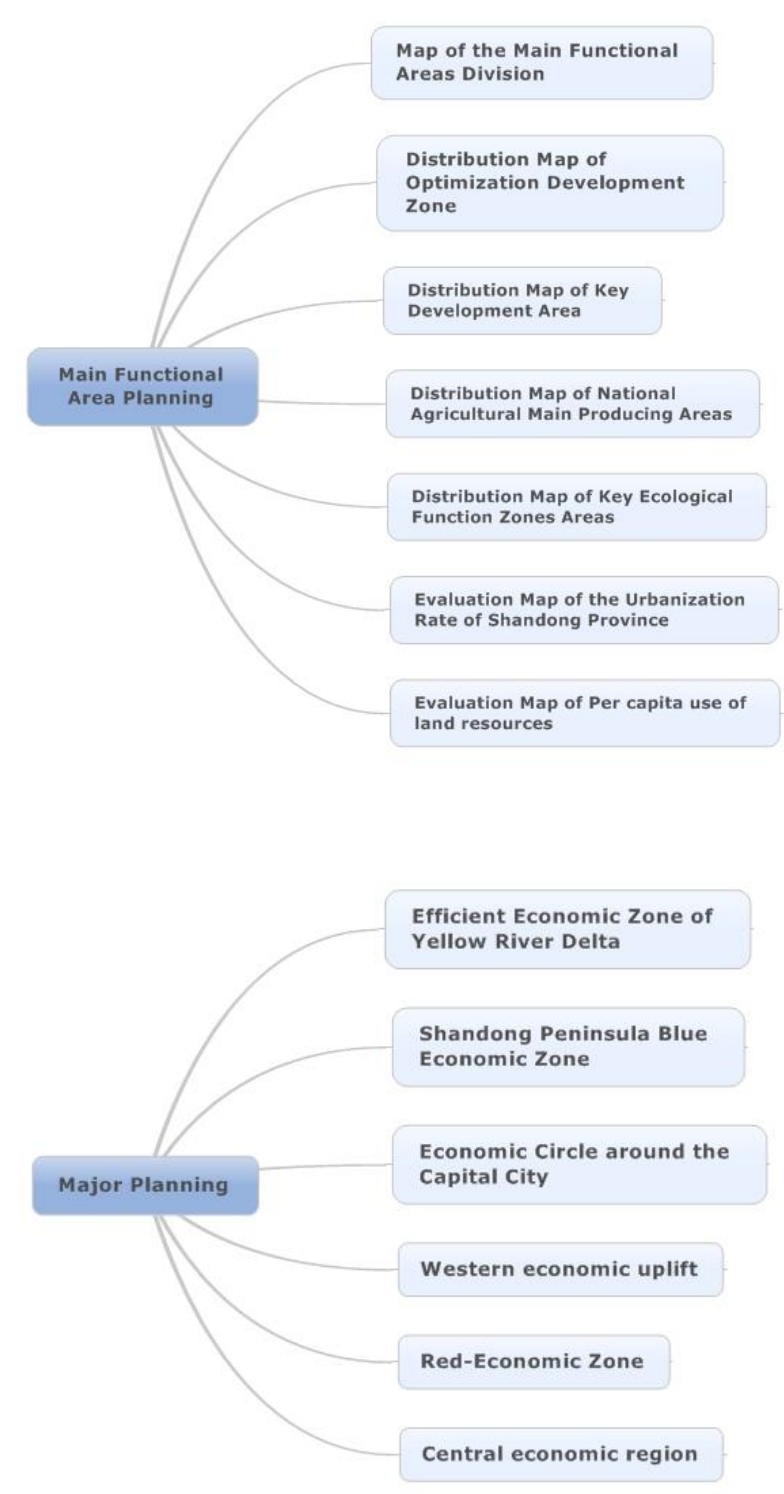

Figure 1. The four main types of statistical charts and maps in Shandong General Provincial Situation Visualization System Based on iOS

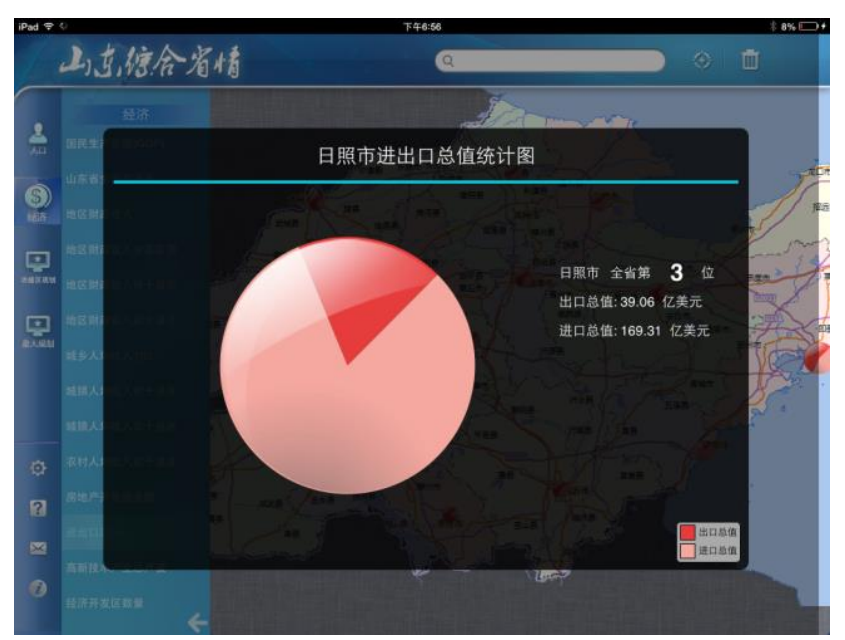

Figure 2. input and export value of 17 cities of Shandong province

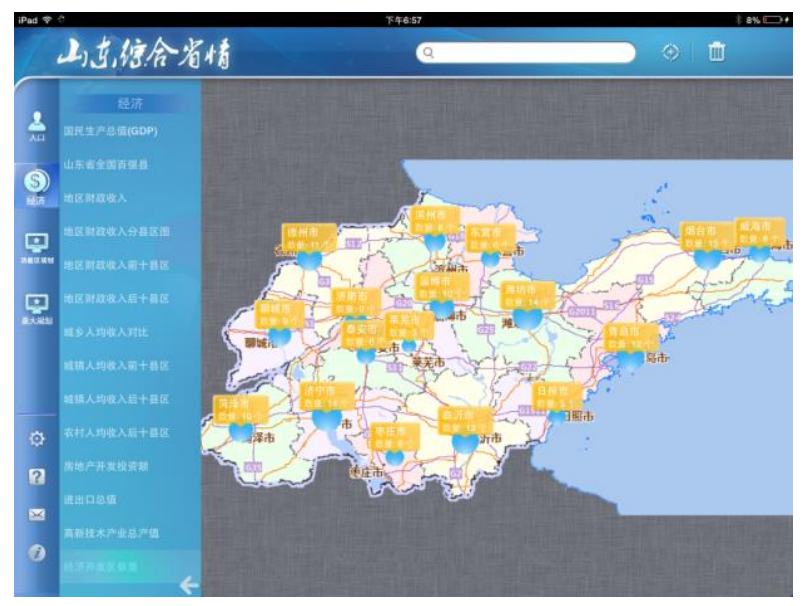

Figure 3. The number of Economic Development Zones in Shandong Province

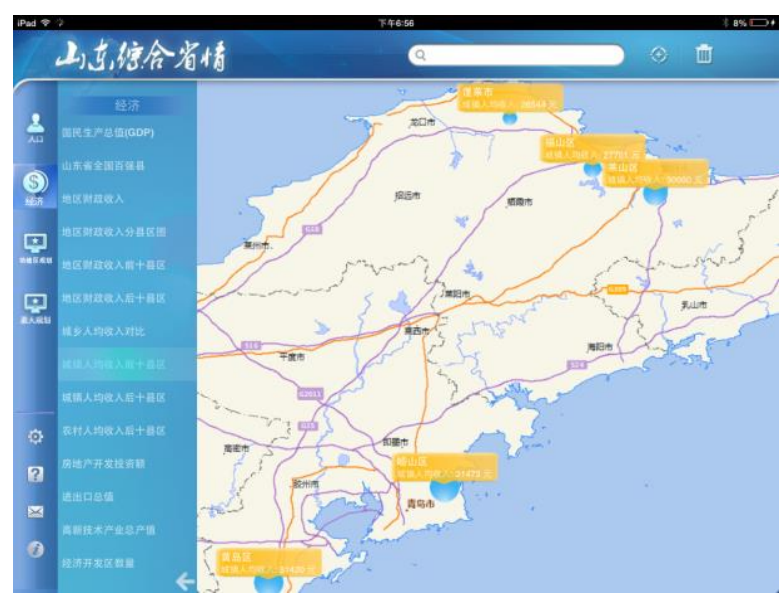

Figure 4. the Top 10 Counties of the Per Capita Income of urban residents in Shandong Province

In the recent years, a series of main planning is been designed and carried forward in Shandong province. Therefore, the system specially included 13 maps of main function area planning included the main functional areas division, optimization development zone, the key development area, the national agricultural main producing areas, the key ecological function zones areas, the urbanization rate of Shandong province and the per capita use of land resources (Figure 5) and five maps of main planning which often been mentioned in the economic planning. 


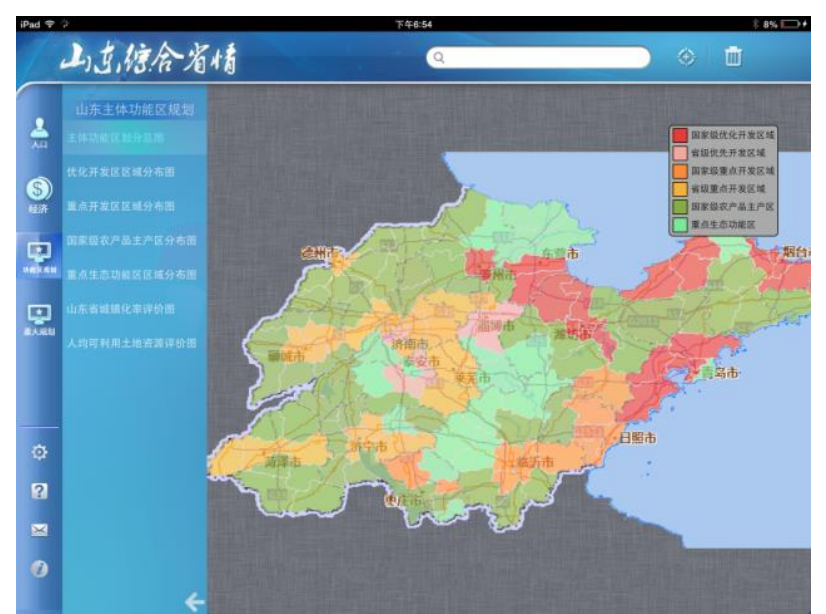

Figure 5. The Map of Main Functional Areas Division of Shandong Province

\section{CONCLUSION}

The system tries to figure out the optimal solution in presenting the general provincial situation information considering of its multi-scale, multi-temporal and multi-dimensional feature with the statistics and thematic map. The General Provincial Situation Visualization System of Shandong province is designed with the Apple's iOS style, and supports the iPad gestures and user habits with a flexible layout to optimize the user interaction and experience. With all this effort, the system implements the visual expression and comprehensive analysis of the general provincial situation information of Shandong province, and provides a professional information support to the government departments and research institutions to understanding the general development situation and planning the significant projects within the Shandong province. The system is currently available for download in the Appstore and is chosen as the typical application case of ESRI China ArcGIS API for iOS.

\section{References from Journals:}

Chen Sheng, Zeng Xingji, 2008. Study on visualization of statistics data based on GIS. Computer Engineering and Design, 29 (14),pp.3757-3759,3762.

Tian feng, Wen Lirong, 2009. The research on visualization of Liaoning provincial comprehensive information. Geomatics and Spatial Information Technology, 32 (6),pp.84-86.

Su Ying, Wang Yingjie, 2005. The design and research on geovisualization system for population. Science of Surveying and Mapping,30 (3)pp. 38-40.

An Kai, Xiao Yu, 2012. A functional zoning visualization system for sustainable development in China. Resources Science, 34 (9)pp.1664-1470.

McCormick B H, Defanti T A, Brown M D, 1987. Visualization in scientific computing, Computer Graphics, 21(6):1-14.

Keim D A,2002. Information visualization and visual data mining. IEEE Trans, Visualization and Computer Graphics, 8(1):1-8.

\section{References from Books:}

Groot R, Mclaughlin J, 2000. Geospatial Data Infrastructure: Concepts, Cases and Good Practice. New York: Oxford University Press.

Marco D, Jennings M,2004. Univeral Meta Data Models. Indianapolis: Wiley Publishing Inc.,.

\section{References from Other Literature:}

The specifications of service release and registration Interface of the 'World-map Shandong', 2012.Land Surveying and Mapping Institute of Shandong Province. Jinan, China. 